\title{
Técnica de Interpolação Direta de Elementos de Contorno aplicada a um Modelo Advectivo-Difusivo com Campo de Velocidade Variável Incompressível
}

\author{
Vitor Pancieri Pinheiro ${ }^{1}$ \\ PPGEM - Programa de Pós-Graduação em Engenharia Mecânica, Universidade Federal de Espírito Santo, \\ ES, Brasil. \\ CCTMEC - Coordenadoria do Curso Técnico em Mecânica, Instituto Federal do Espírito Santo -IFES, \\ Campus Guarapari, ES, Brasil. \\ Carlos Friedrich Loeffler ${ }^{2}$ \\ PPGEM - Programa de Pós-Graduação em Engenharia Mecânica, Universidade Federal de Espírito Santo, \\ ES, Brasil. \\ Natan Sian das Neves ${ }^{3}$ \\ COPPE/UFRJ - Instituto Alberto Luiz Coimbra de Pós-Graduação e Pesquisa de Engenharia, Programa \\ de Engenharia Civil, Universidade Federal do Rio de Janeiro, RJ, Brasil. \\ Larissa Maciel de Almeida ${ }^{4}$ \\ CIM -Analista de Dados - Autoglass, Vila Velha, ES
}

\begin{abstract}
Resumo.
A abordagem de problemas advectivo-difusivos por variantes do método de elementos de contorno (MEC) focadas em aproximação por funções de bases radiais tal como a formulação da interpolação direta (MECID) e a clássica formulação de dupla reciprocidade (MECDR) ainda permanecem um obstáculo relevante ao método. A intensificação dos campos hidrodinâmicos neste tipo de modelo gera efeitos de advecção dominante e de compressibilidade, que por sua vez, dificultam de sobremaneira a performance de qualquer formulação discreta. Neste artigo, examina-se de forma isolada a influência da advecção na precisão da técnica MECID através de um problema-teste bidimensional com campo de velocidade variável, entretanto com escoamento incompressível. De forma geral os resultados obtidos são precisos e estáveis para o intervalo de Péclet global testado e demonstram uma maior robustez numérica frente aos efeitos advectivos frente a técnica da dupla reciprocidade.
\end{abstract}

Palavras-chave. Método de Elementos de Contorno, Técnica de Interpolação Direta, Técnica da Dupla Reciprocidade, Modelo Advectivo-Difusivo, Efeitos Advectivos.

\section{Introdução}

A solução de modelos advectivo-difusivos constitui tarefa desafiadora para uma ampla gama de técnicas numéricas disponíveis. Tal fato pode ser em muito explicado pela natureza híbrida deste tipo de modelo matemático, que pode se comportar de maneira parabólica em situações física

\footnotetext{
${ }^{1}$ vitor.pinheiro1987@gmail.com

2loefflercarlos@gmail.com

3 natan.sian@gmail.com

${ }^{4}$ larissa.m.de.almeida@gmail.com
} 
dominadas pelos processo de difusão ou de forma hiperbólica em casos dominados pelos efeitos convectivos $[8,10]$. O surgimento de formulações mais versáteis do método de elementos de contorno (MEC), baseadas em aproximações por bases radiais, tal como a técnica da dupla reciprocidade (MECDR) [5] e a mais recente técnica da interpolação direta [3] (MECID) trouxeram enorme versatibilidade e robustez de aplicação ao método, já que utilizam a solução fundamental de Laplace no lugar da solução fundamental correlata ao modelo específico. No escopo de problemas advectivodifusivos, entretanto, as duas técnicas enfrentam desafios em termos de precisão a medida que os efeitos advectivos tornam-se dominantes. A dupla reciprocidade mostra-se eficiente apenas em situações de baixo número de Péclet [10] em modelos advectivo-difusivos de velocidade constante, quando aplicada de forma isolada. Em contrapartida, recentemente, Loeffler e Pinheiro [6] testaram a técnica da interpolação direta em modelos advectivo-difusivos com campo de velocidade uniforme, onde a mesma já mostrou-se precisa e robusta para um intervalo mais amplo de números de Péclet.

Em casos de velocidade variável, propostas que usam a decomposição do campo de velocidade como uma média adicionada de uma flutuação, respectivamente abordadas pela formulação clássica e pela dupla reciprocidade, apresentam desempenho satisfatório para Péclet globais de baixos a altos $[1,10]$. Entretanto, a aplicação isolada da formulação da técnica da dupla reciprocidade em problemas advectivos difusivos é escassa na literatura tanto para campos uniformes de velocidade quanto para campos variáveis o que cria uma maior demanda por comparar a performance da mesma frente a outras técnicas baseadas em aproximações por bases radiais como a MECID, por exemplo. Tais comparações, no tocante à MECID já vem sendo feitas de forma preliminar [7], entretanto, um paralelo mais amplo e sistêmico compõe claramente uma lacuna na literatura.

Neste artigo realiza-se um comparativo entre as técnica MECDR e MECID em um caso um problema advectivo-difusivo com velocidade variável, entretanto, com escoamento incompressível. Tal simplificação hidrodinâmica ressalta os dois efeitos físicos desafiadores para as formulações baseadas em bases radiais: efeitos de convecção dominante e efeitos de compressibilidade. No caso, à obediência a hipótese de incompressibilidade permite uma análise exclusiva dos efeitos de advecção sobre a precisão das técnicas analisadas.

\section{Modelagem Matemática}

A descrição em termos matemáticos de um problema de campo escalar do tipo advectivodifusivo passa, de forma geral, pela representação de balanços físicos gerais [9], que neste caso, resumem-se a conservação de massa e energia, e dados por (1) e (2).

$$
\frac{D \varrho}{D t}+\varrho \nabla \cdot \vec{v}=0,
$$

Onde as variáveis de interesse são o campo de massa específica $\varrho$, campo de velocidade do escoamento $\vec{v}$, campo de pressão $p$, campo de temperatura $T, \Phi$ o termo de dissipação viscosa, $c_{p}$ o calor específico a pressão constante, $\lambda$ o coeficiente de condutividade térmica e $\beta$ contabilizando o coeficiente de expansão volumétrica.

$$
\varrho c_{p} \frac{D T}{D t}=\operatorname{div}(\lambda \nabla T)+\beta T \frac{D p}{D t}+\Phi .
$$

As hipóteses simplificadoras implícitas que nos permitem escrever as leis conservativas nas formas acima incluem a modelagem de um fluido como contínuo, simetria dos tensor das tensões do fluido, isotropia e reologia newtoniana do fluido, validade da hipótese de Stokes, não consideração de fontes e sumidouros no balanço de energia e também adoção da lei de Fourier como modelo representativo da taxa de calor por mecanismo condutivo. 
Para a simplificação do balanço de massa geral, dado por (1), adota-se ainda a hipótese de incompressibilidade do fluido, que pode ser definido conceitualmente com a nulidade da derivada total do campo de massa específica do escoamento, algebricamente representada por (3). Em adição a este conceito, se a massa específica puder ser considerada constante em todo o domínio computacional, a consequência algébrica direta desta hipótese é a nulidade do divergente do campo de velocidade também mostrada em (3).

$$
\frac{D \varrho}{D t}=0 \quad \therefore \quad \nabla \cdot \vec{v}=0 .
$$

Para adequar agora a escrita da equação de balanço energético, equação (2), aplica-se as seguintes hipóteses simplificadoras: abordagem bidimensional, propriedades termodinâmicas constantes, dissipação viscosa desprezível e regime permanente. O que resulta em:

$$
\varrho c_{p}\left(u \frac{\partial T}{\partial x}+v \frac{\partial T}{\partial y}\right)=\lambda\left[\frac{\partial^{2} T}{\partial x^{2}}+\frac{\partial^{2} T}{\partial y^{2}}\right] .
$$

A estrutura matemática em (4) é conhecida como modelo de advecção-difusão, sendo capaz de representar problemas de natureza térmica, dispersão de poluentes dentre outros fenômenos de interesses em engenharia. Esta é a equação de governo a ser solucionada numericamente neste artigo.

\section{Técnica da Interpolação Direta}

No intuito de melhor representar a formulação integral e conceito central da técnica de interpolação direta, opta-se por trabalhar em notação indicial, devido a sua sinteticidade e elegância. A equação de governo é representada nesta notação a seguir, em (5), onde o campo escalar de temperatura em (4), foi substituído por um campo físico escalar genérico denotado por $u$ e a difusividade térmica domínio representada por $\alpha$.

$$
\alpha u_{, i i}=v_{i} u_{, i}, \quad \alpha=\frac{\lambda}{\varrho c_{p}} .
$$

O tratamento matemático integral da equação de governo (5), portanto, pode ser agora dividido em duas grandes partes: o lado difusivo (LD), lado esquerdo da equação e o lado direito que contabiliza os efeitos advectivos (LA). O lado difusivo, possui formulação integral inversa e seu tratamento integral é vastamente difundido, conforme pode ser apreciado na literatura [2] e representado algebricamente abaixo:

$$
L D=c(\xi) u(\xi)+\int_{\Gamma} u(X) q^{*}(\xi, X) d \Gamma-\int_{\Gamma} q(X) u^{*}(\xi, X) d \Gamma
$$

O lado advectivo possui um tratamento ligeiramente mais extenso, e maiores detalhamentos podem ser encontrados nas referências [6] e [7]. Todavia, com uso recorrente de integração por partes e também do teorema de divergência, para representar algumas integrais de domínio em termos do contorno, pode-se, depois de certa álgebra, alcançar a seguinte formulação integral final do lado advectivo (LA). 


$$
\begin{aligned}
& L A=\int_{\Gamma} n_{i}(X) v_{i}(X) u(X) u^{*}(\xi ; X) d \Gamma-u(\xi) \int_{\Gamma} n_{i}(X) v_{i}(X) u(X) u^{*}(\xi ; X) d \Gamma \\
& +\int_{\Omega} v_{i}(X) u_{, i}^{*}(\xi ; X)[u(X)-u(\xi)] d \Omega-\int_{\Omega} v_{i, i}(X) u^{*}(\xi ; X)[u(X)-u(\xi)] d \Omega .
\end{aligned}
$$

O formato integral final do lado advectivo, em (7), representa um tratamento amplo para problemas de velocidade variável via técnica de interpolação direta e alguns detalhes relevantes podem serem observados. Primeiramente, na estrutura geral, percebe-se que as duas primeiras integrais já forma conduzidas a uma escrita de contorno, enquanto as duas últimas integrais de domínio são justamente os alvos das aproximação por bases radiais a porvir. Ainda nas integrais de domínio, observa-se a diferença entre o potencial do ponto campo $X$ e do ponto fonte $\xi$ no núcleo das integrais, o que indica que o procedimento de regularização, implícito a técnica de interpolação direta já foi utilizado e cujos detalhes algébricos encontram-se em [3]. Por fim, mas não menos importante, observa-se que a primeira integral de domínio é escrita com núcleo dependente do campo de velocidade do problema, enquanto a segunda depende do divergente deste mesmo campo. Pode-se dizer que os efeitos advectivos estão contabilizados pela primeira, enquanto efeitos de compressibilidade estão implícitos na valoração na segunda. No problema abordado neste artigo, a última integral é nula, e temos portanto, uma análise exclusiva dos efeitos da advecção.

A técnica de interpolação direta, por sua vez, tem como proposta central aproximar toda a completude dos núcleos das integrais de domínio remanescentes em (7), em contraste com a técnica MECDR, que aproxima apenas parcialmente o núcleo citado. Ao aplicar a técnica na primeira integral de domínio em (7), inerente aos efeitos advectivos, tem-se em (8):

$$
v_{i}(X) u_{i}^{*}(\xi, X)[u(X)-u(\xi)] \cong \alpha_{j}^{\xi} F_{j}\left(X_{j}, X\right) .
$$

A seleção de funções de bases radiais, em termos de precisão, para problemas relevantes de campo escalar é um tópico de pesquisa ativo e um maior volume de informações sobre funções de bases radiais de amplo uso e sua interação com a técnica MECID, podem ser encontradas em [4].

\section{Experimento Numérico}

Nesta seção um problema de modelo é testado para inferir a performance da técnica MECID. Entretanto, antes de ressaltar mais detalhes, é preciso estabelecer certas diretrizes de metodologia. O domínio é caracterizado por uma geometria quadrada e a malha computacional é formada por elementos de contorno com interpolação linear e pólos internos no domínio, conforme sintetizado abaixo na Figura 1.

Observa-se na Figura 1 problema teste que consiste de uma domínio computacional quadrado exposto a condições de contorno de Dirichlet e a um campo de velocidade que aumenta linearmente ao longo das arestas. Como a mesma condição funcional é imposta como condição nas quatro arestas a solução analítica do problema é automática. Este problema possui uma simetria diagonal, e os resultados são considerados como uma média entre os nós das arestas inferior e direita.

Quanto a malha computacional, pode-se distinguir, também na Figura 1, entre nós de contorno que formam os elementos lineares, nós duplos, nós que compõem a primeira fileira de pontos internos, também pontos internos a partir da primeira fileira, de acordo com a legenda. As interpolações da aproximação do MECID, dada por (8), são feita utilizando função de base radial de placa fina. A variável $\alpha$, definida também no esquema da Figura 1, representa a proporção de aproximação da primeira fileira de internos em relação ao contorno, tendo valor unitário para o caso de malha estruturada e diminuindo com a aproximação da fileira ao contorno do domínio. 

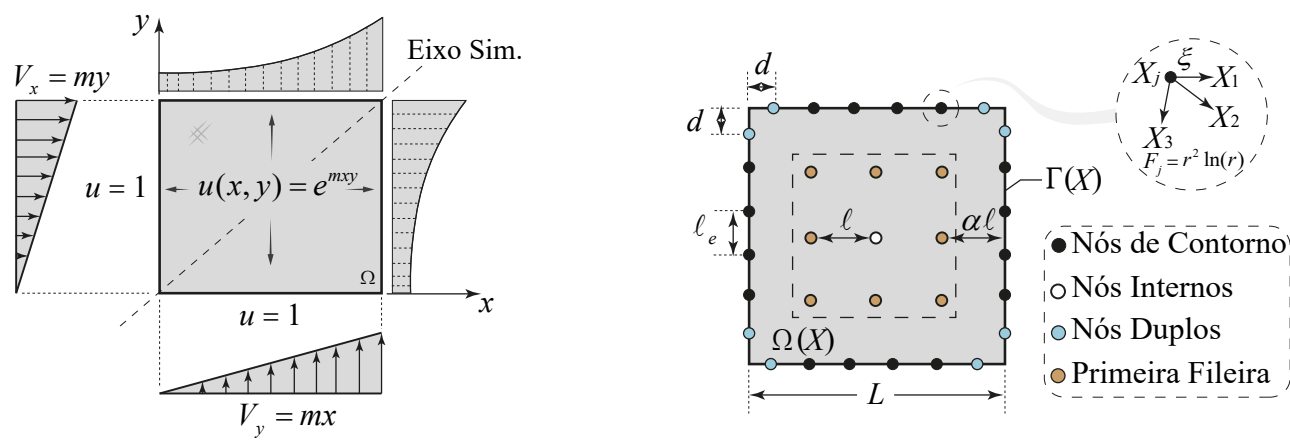

Figura 1: (a) Domínio Computacional e (b) Malha Numérica

A geração de resultados do caso em análise inicia pela exposição dos testes de convergência em relação aos dois principais parâmetros de refinamento de malha: quantidade de elementos de contorno e quantidade e polos internos interpolantes. Na sequência uma investigação do parâmetro $\alpha$ é performada para inferir sobre a sensibilidade da formulação MECID ao posicionamento da primeira fileira de internos, e ademais, se há um posicionamento mais favorável, no caso em análise. Por fim, a análise principal consiste em incrementar a intensidade do campo de velocidade e consequentemente o número de Péclet, e mensurar a precisão da técnica MECID comparativamente à MECDR, em dois níveis de refinamento de malha.

A convergência da técnica é analisada nos dois gráficos que seguem da Figura 2a e 2b, em relação a quantidade de elementos na discretização do contorno e polos internos, respectivamente.

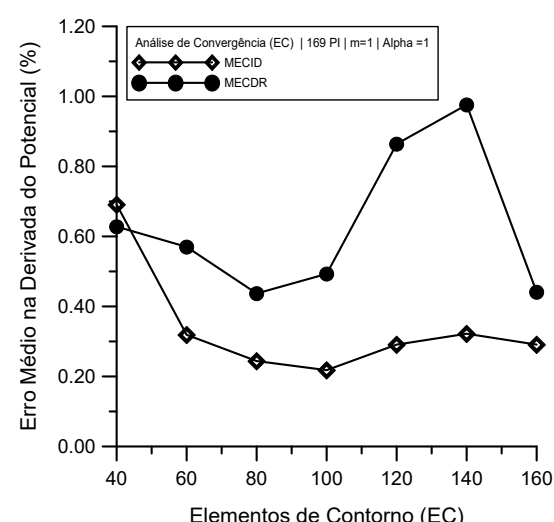

(a)

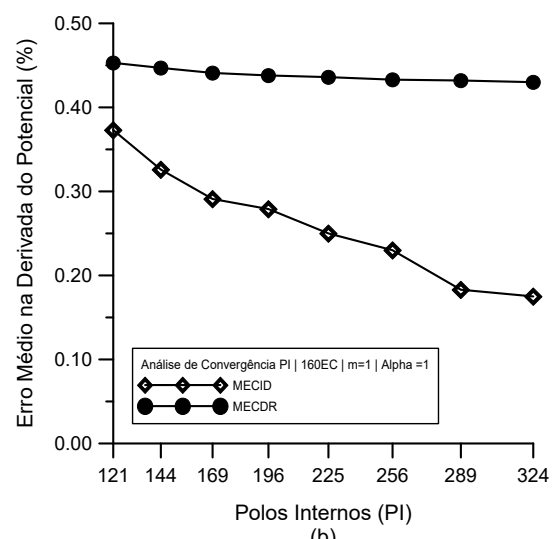

(b)

Figura 2: Convergência de Malha: (a) Elementos de Contorno (b) Polos Internos

Quanto ao refinamento do contorno, observa-se na Figura 2a um comportamento não monotônico em ambas as formulações numéricas testadas, o que provavelmente deve-se ao campo de velocidade variável do problema teste. Já em relação aos polos internos, na Figura $2 \mathrm{~b}$, há um alinhamento com comportamento já esperado, onde a MECID mostra-se mais sensível ao refinamento deste parâmetro e a MECDR, praticamente invariante, em termos de nível de erro médio.

O posicionamento da primeira fileira de pontos internos é testada e os resultados expostos na Figura 3a e 3b, para a MECID e para a MECDR, respectivamente. A técnica MECID mostra-se bastante sensível ao parâmetro $\alpha$ para os três níveis de malhas testados. Já a técnica da dupla reciprocidade mostrou-se praticamente não afetada por este parâmetro, como já era esperado. 


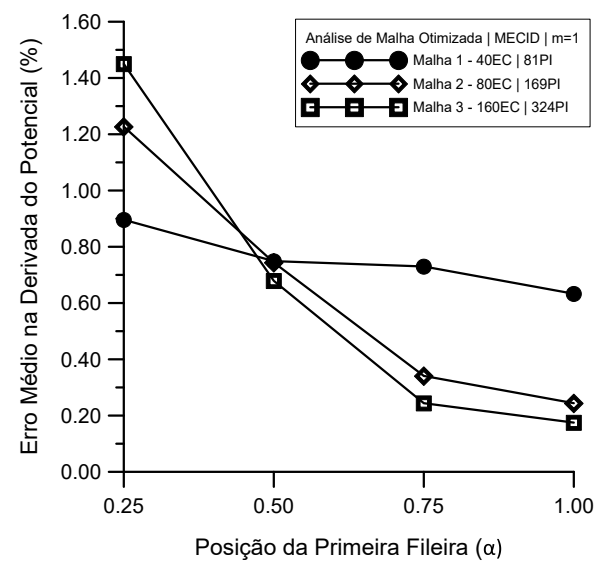

(a)

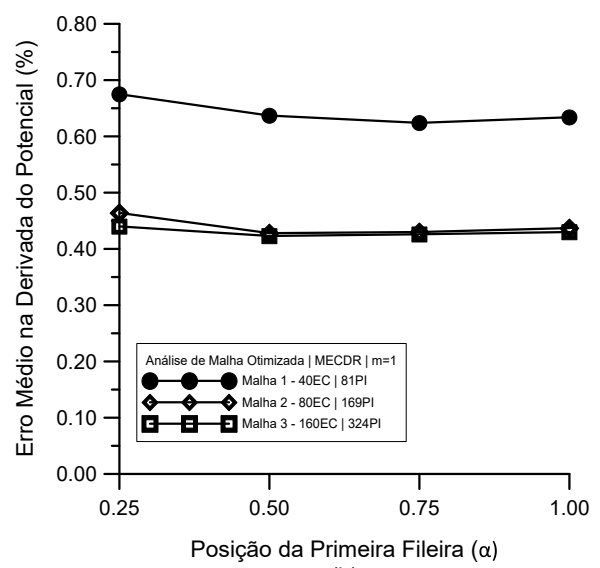

(b)

Figura 3: Posição da Primeira Fileira (a) MECID (b) MECDR

Na sequência, executa-se uma análise paramétrica em função de $m$ e compara-se a performance do MECID em paralelo ao MECDR frente a um aumento gradual dos efeitos advectivos. Os resultados das análises são mostrados abaixo na Figura 4, no gráfico (a) para uma malha de refinamento mediano e uma malha mais refinada no gráfico (b).

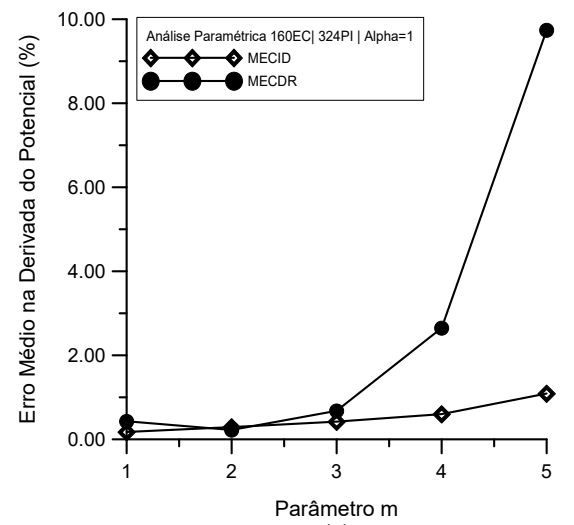

(a)

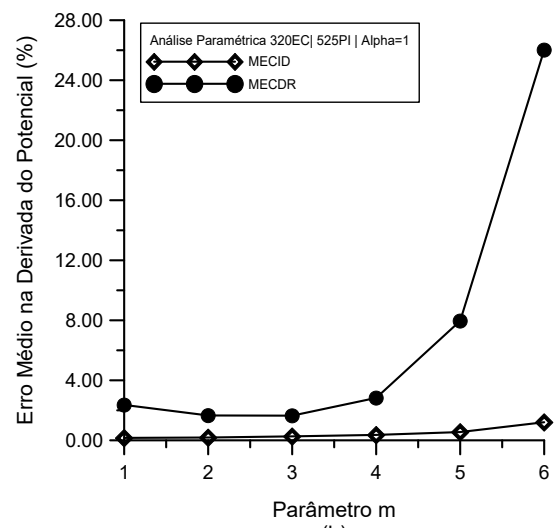

(b)

Figura 4: Efeitos Advectivos - Parâmetro m : (a) Malha Intermediária (b) Malha Refinada

Na malha intermediária da Figura 4a é possível perceber que os erros médios apresentados pelas duas técnicas são muito parelhos até $m=3$. Para valores subsequentes de $m$ a técnica MECDR perde muita precisão gerando erros na caso de $10 \%$ para o último valor testado do parâmetro. Já a técnica MECID mantém precisão satisfatória com erros inferiores a $2 \%$ em todo intervalor testado. Por fim, para a malha mais refinada, Figura 4b, os os comportamentos gerais se mantém, com erros significantes para a MECDR com parâmetro $m$ maior que 3 e precisão satisfatória da técnica MECID para todo o intervalo testado do parâmetro, com sensível melhora nos níveis de erro com o refinamento de malha, com erros médios abaixo de $1 \%$ em todas as simulações performadas. Ainda no tocante a técnica MECDR, o aumento de nível de erros médios para valores fixados do parêmtro $m$, com o refinamento de malha, revela problemas de mau condicionamento matricial, que não se apresentam nos resultados do MECID. 


\section{Conclusão}

Os testes performados constataram de forma reinterada uma maior sensibilidade da técnica de interpolação direta em relação a quantidade de polos internos e seu posicionamento contra uma sensibilidade tímida da dupla reciprocidade em relação a tais parâmetros. Este fato corrobora para a robustez da técnica MECID, que possui elementos de contorno e polos internos como parâmetros efetivos de refinamento de malha. O comparativo das duas técnicas no contexto de incremento gradual dos efeitos advectivos aponta para uma maior resiliência de precisão por parte da interpolação direta, no caso testado. A dupla reciprocidade, mesmo para baixos números de Péclet já mostra-se incapaz de representar o fenômeno físico posto. Desta maneira testes mais sistêmicos que envolvam comparações entre estas duas técnicas focadas em bases radiais, em problemas de velocidade variável são necessárias, uma vez que, a presença de efeitos de compressibilidade para além dos efeitos advectivos pode alterar sensivelmente a performance relativa entre as formulações.

\section{Referências}

[1] AL-Bayati, S. A., Wrobel, L. C. (2018). The dual reciprocity boundary element formulation for convection-diffusion-reaction problems with variable velocity field using different radial basis functions. International Journal of Mechanical Sciences, 145, 367-377.

[2] Brebbia, C. A., Dominguez, J. (1994). Boundary elements: an introductory course. WIT press.

[3] Loeffler, C. F., Mansur, W. J. (2017). A regularization scheme applied to the direct interpolation boundary element technique with radial basis functions for solving eigenvalue problem. Engineering Analysis with Boundary Elements, 74, 14-18.

[4] Loeffler, C. F., Zamprogno, L., Mansur, W. J., Bulcão, A. (2017). Performance of compact radial basis functions in the direct interpolation boundary element method for solving potential problems. Computer Modeling in Engineering Sciences, 113(3), 367-387.

[5] Nardini, D., Brebbia, C. A. (1983). A new approach to free vibration analysis using boundary elements. Applied mathematical modelling, 7(3), 157-162.

[6] Pinheiro, V.P. (2018) Application of the Boundary Element Method with Regularized Direct Integration to Two-dimensional Advective-Diffusive Problems. Master's Thesis (in portuguese). Universidade Federal do Espírito Santo.

[7] Pinheiro, V.P., Loeffler, C.F., Almeida, L.M. (2020) A Brief Performance Analysis of Direct Integration Technique applied on Bidimensional Advective-Diffusive Problems with Variable Velocity Fields. Proceedings of the XLI Ibero-Latin-American Congress on Computational Methods in Engineering, CILAMCE 2020, Foz do Iguaçu/PR, Brazil.

[8] Qiu, Z. H., Wrobel, L. C., Power, H. (1998). Numerical solution of convection-diffusion problems at high Péclet number using boundary elements. International Journal for Numerical Methods in Engineering, 41(5), 899-914.

[9] Schlichting, H.,Gersten, K. (2016). Boundary-layer theory. Springer.

[10] Wrobel, L. C., DeFigueiredo, D. B. (1991). A dual reciprocity boundary element formulation for convection-diffusion problems with variable velocity fields. Engineering Analysis with Boundary Elements, 8(6), 312-319. 